\title{
Naimark-Sacker bifurcation of second order rational difference equation with quadratic terms
}

\author{
M. R. S. Kulenovića , S. Moranjkićb ${ }^{\text {, Z. Nurkanović }}{ }^{b, *}$ \\ ${ }^{a}$ Department of Mathematics, University of Rhode Island, Kingston, RI 02881, USA. \\ ${ }^{b}$ Department of Mathematics, University of Tuzla, 75350 Tuzla, Bosnia and Herzegovina.
}

Communicated by R. Saadati

\begin{abstract}
We investigate the global asymptotic stability and Naimark-Sacker bifurcation of the difference equation

$$
x_{n+1}=\frac{F}{b x_{n} x_{n-1}+c x_{n-1}^{2}+f}, \quad n=0,1, \cdots,
$$

with non-negative parameters and nonnegative initial conditions $x_{-1}, x_{0}$ such that $b x_{0} x_{-1}+c x_{-1}^{2}+f>0$. By using fixed point theorem for monotone maps we find the region of parameters where the unique equilibrium is globally asymptotically stable. (C)2017 All rights reserved.
\end{abstract}

Keywords: Attractivity, bifurcation, difference equation, invariant, Naimark-Sacker bifurcation, periodic solution. 2010 MSC: 39A10, 39A28, 39A30.

\section{Introduction and preliminaries}

In this paper we investigate the global dynamics of the following difference equation

$$
x_{n+1}=\frac{F}{b x_{n} x_{n-1}+c x_{n-1}^{2}+f}=H\left(x_{n}, x_{n-1}\right), \quad n=0,1, \cdots
$$

where

$$
F, b, c, f \in(0, \infty),
$$

and the initial conditions $x_{-1}$ and $x_{0}$ are arbitrary nonnegative real numbers. Equation (1.1) is the special case of a general second order quadratic fractional equation of the form

$$
x_{n+1}=\frac{A x_{n}^{2}+B x_{n} x_{n-1}+C x_{n-1}^{2}+D x_{n}+E x_{n-1}+F}{a x_{n}^{2}+b x_{n} x_{n-1}+c x_{n-1}^{2}+d x_{n}+e x_{n-1}+f}, \quad n=0,1, \ldots,
$$

\footnotetext{
*Corresponding author

Email addresses: mkulenovic@mail.uri.edu (M. R. S. Kulenović), samra.moranjkic@untz.ba (S. Moranjkić), zehra.nurkanovic@untz.ba (Z. Nurkanović)

doi:10.22436/jnsa.010.07.11
} 
with nonnegative parameters and initial conditions such that $A+B+C>0, a+b+c+d+e+f>0$ and $a x_{n}^{2}+b x_{n} x_{n-1}+c x_{n-1}^{2}+d x_{n}+e x_{n-1}+f>0, n=0,1, \cdots$. Several global asymptotic results for some special cases of (1.2) were obtained in $[4-6,15]$. The systematic theory of the linear fractional difference equation

$$
x_{n+1}=\frac{D x_{n}+E x_{n-1}+F}{d x_{n}+e x_{n-1}+f}, \quad n=0,1, \cdots,
$$

with nonnegative parameters and initial conditions such that $D+E+F>0, d+e+f>0$ and

$$
d x_{n}+e x_{n-1}+f>0, \quad n=0,1, \cdots,
$$

was presented in [7] where it was shown that (1.3) does not exhibit Naimark-Sacker bifurcation and can only exhibit either conservative chaos or period doubling bifurcation, see $[7,10,11]$. In the case of quadratic fractional difference equation (1.2) we showed that Naimark-Sacker bifurcation is very common, see [12]. First systematic study of global dynamics of a special case of (1.2) where $A=C=D=a=c=$ $\mathrm{d}=0$ was performed in $[1,2]$.

The global attractivity result in [7,9], which is the fixed point theorem for monotone maps that will be used here is the following result.

Theorem 1.1. Assume that the difference equation

$$
x_{n+1}=G\left(x_{n}, \ldots, x_{n-k}\right), \quad n=0,1, \ldots,
$$

where $\mathrm{G}$ is nondecreasing functions in all its arguments has the unique equilibrium $\bar{x} \in \mathrm{I}$, where $\mathrm{I}$ is an invariant interval, that is $\mathrm{G}: \mathrm{I}^{\mathrm{k}+1} \rightarrow \mathrm{I}$. Then $\overline{\mathrm{x}}$ is globally asymptotically stable.

In this paper we perform the local stability analysis of the unique equilibrium and give the necessary and sufficient conditions for the equilibrium to be locally asymptotically stable, a repeller or a non-hyperbolic equilibrium. The local stability analysis indicates that some possible dynamic scenarios for (1.1) include Naimark-Sacker bifurcation. We apply Theorem 1.1 in the part of the region of local asymptotic stability to obtain global asymptotic stability result. In the complement of the parametric region where the equilibrium is locally stable the equilibrium becomes repeller with two characteristic values to be complex conjugate numbers and on the boundary of this region two characteristic values are complex conjugate numbers on the unit circle. We show that in this case (1.1) exhibits Naimark-Sacker bifurcation resulting in the existence of locally stable periodic solution of unknown period.

\section{Linearized stability analysis}

In this section we present the local stability of the unique positive equilibrium of (1.1).

In view of the above restriction on the initial conditions of (1.1), the equilibrium points of (1.1) are the positive solutions of the equation

or equivalently

$$
\bar{x}=\frac{F}{(b+c) \bar{x}^{2}+f^{\prime}}
$$

$$
(b+c) \bar{x}^{3}+f \bar{x}-F=0 .
$$

By Descartes rule of sign (2.1) has the unique positive solution $\bar{x}$ given as

$$
\bar{x}=\sqrt[3]{\frac{1}{2(b+c)}\left(F+\sqrt{F^{2}+\frac{4 f^{3}}{27(b+c)}}\right)}-\frac{f}{3(b+c) \sqrt[3]{\frac{1}{2(b+c)}\left(F+\sqrt{F^{2}+\frac{4 f^{3}}{27(b+c)}}\right.}} .
$$

Now we investigate the stability of the positive equilibrium of (1.1). Set

and observe that

$$
H(u, v)=\frac{F}{b u v+c v^{2}+f^{\prime}}
$$




$$
\mathrm{H}_{u}^{\prime}(\mathrm{u}, v)=\frac{-b F v}{\left(b u v+c v^{2}+f\right)^{2}}, \quad H_{v}^{\prime}(u, v)=\frac{-F(b u+2 c v)}{\left(b u v+c v^{2}+f\right)^{2}}
$$

If $\bar{x}$ denotes an equilibrium point of (1.1), then the linearized equation associated with (1.1) about the equilibrium point $\bar{x}$ is

$$
z_{\mathrm{n}+1}=\mathrm{s} z_{\mathrm{n}}+\mathrm{t} z_{\mathrm{n}-1}
$$

where

$$
\mathrm{s}=\mathrm{H}_{\mathfrak{u}}^{\prime}(\overline{\mathrm{x}}, \overline{\mathrm{x}}) \quad \text { and } \quad \mathrm{t}=\mathrm{H}_{v}^{\prime}(\overline{\mathrm{x}}, \overline{\mathrm{x}}) .
$$

Theorem 2.1. The unique equilibrium point $\bar{x}$ of (1.1) given by (2.2) is

(i) locally asymptotically stable if $\mathrm{f}^{3}>\frac{\mathrm{c}^{3} \mathrm{~F}^{2}}{(\mathrm{~b}+2 \mathrm{c})^{2}}$;

(ii) a repeller if $\mathrm{f}^{3}<\frac{\mathrm{c}^{3} \mathrm{~F}^{2}}{(\mathrm{~b}+2 \mathrm{c})^{2}}$;

(iii) a non-hyperbolic point of elliptic type if $\mathrm{f}^{3}=\frac{\mathrm{c}^{3} \mathrm{~F}^{2}}{(\mathrm{~b}+2 \mathrm{c})^{2}}$.

Proof. A straightforward calculation yields

$$
s=H_{u}^{\prime}(\bar{x}, \bar{x})=\frac{-b F \bar{x}}{\left((b+c) \bar{x}^{2}+f\right)^{2}}=\frac{-b \bar{x}^{3}}{F}<0,
$$

and

$$
t=H_{v}^{\prime}(\bar{x}, \bar{x})=\frac{-F(b+2 c) \bar{x}}{\left((b+c) \bar{x}^{2}+f\right)^{2}}=\frac{-(b+2 c) \bar{x}^{3}}{F}<0 .
$$

Thus $s-t>0$ and

$$
s^{2}-(1-t)^{2}=(s+t-1)(s-t+1)<0,
$$

i.e.,

$$
|s|<|1-t| .
$$

The unique equilibrium point $\bar{x}$ is a non-hyperbolic point of elliptic type for $t=-1$. Eigenvalues are given by

$$
\lambda_{1,2}=\frac{-b \pm i \sqrt{4(b+2 c)^{2}-b^{2}}}{2(b+2 c)},
$$

and so $\left|\lambda_{1,2}\right|=1$. Hence, for

$$
t=-1 \Leftrightarrow \frac{-(b+2 c) \bar{x}^{3}}{F}=-1 \Leftrightarrow \bar{x}=\sqrt[3]{\frac{F}{b+2 c}} .
$$

Thus

so

$$
(b+c) \frac{F}{b+2 c}+f \sqrt[3]{\frac{F}{b+2 c}}-F=0 \Leftrightarrow f^{3}=\frac{c^{3} F^{2}}{(b+2 c)^{2}}
$$

$$
\bar{x}=\sqrt[3]{\frac{F}{b+2 c}}=\sqrt{\frac{f}{c}} \text { and } f=c\left(\sqrt[3]{\frac{F}{b+2 c}}\right)^{2} .
$$

Then

$$
s=\frac{-b \bar{x}^{3}}{F}=\frac{-b}{F} \frac{F}{b+2 c}=-\frac{b}{b+2 c}
$$


and

$$
\lambda^{2}+\frac{b}{b+2 c} \lambda+1=0
$$

which solutions are given with (2.3). If $f^{3}>\frac{c^{3} F^{2}}{(b+2 c)^{2}}$ the equilibrium point $\bar{x}$ is locally asymptotically stable $(t \in(-1,0))$, and if $f^{3}<\frac{c^{3} F^{2}}{(b+2 c)^{2}}$ the equilibrium point $\bar{x}$ is a repeller $(t<-1)$. Finally, if $f^{3}=\frac{c^{3} F^{2}}{(b+2 c)^{2}}$, then $\left|\lambda_{1,2}\right|=1$.

\section{Global asymptotic stability}

In this section we give global asymptotic stability result for (1.1). We show that the unique equilibrium point is globally asymptotically stable in the subregion of the parametric region of local asymptotic stability.

Theorem 3.1. The unique equilibrium point $\bar{x}$ of (1.1) is globally asymptotically stable if the following condition holds

$$
f^{3}>\frac{1}{4}(b+c) F^{2} \text {. }
$$

Proof. Every solution of (1.1) satisfies the fourth order difference equation

$$
x_{n+1}=H\left(x_{n}, x_{n-1}\right)=H\left(H\left(x_{n-1}, x_{n-2}\right), H\left(x_{n-2}, x_{n-3}\right)\right)=H_{1}\left(x_{n-1}, x_{n-2}, x_{n-3}\right), \quad n=0,1, \cdots,
$$

where $H_{1}$ is increasing function in all its arguments. Simplifying the right hand side of (3.2) we obtain

$$
x_{n+1}=\frac{F D\left(x_{n-1}, x_{n-2}\right) D\left(x_{n-2}, x_{n-3}\right)^{2}}{b F^{2} D\left(x_{n-2}, x_{n-3}\right)+c F^{2} D\left(x_{n-1}, x_{n-2}\right)+f D\left(x_{n-1}, x_{n-2}\right) D\left(x_{n-2}, x_{n-3}\right)^{2}},
$$

where

$$
\mathrm{D}(\mathrm{u}, \mathrm{v})=\mathrm{buv}+v^{2}+\mathrm{f} .
$$

The equilibrium solution of (3.3) satisfies the equation

$$
\left((b+c) F^{2}+f\left((b+c) x^{2}+f\right)^{2}\right) x-F\left((b+c) x^{2}+f\right)^{2}=0 .
$$

Since the left hand side of (3.4) can be factored as

$$
\left((b+c) x^{3}+f x-F\right)\left((b+c) f x^{2}-(b+c) F x+f^{2}\right),
$$

we conclude that the equilibrium solutions of (3.3) are either equilibrium solutions of (1.1) or the solutions of the quadratic equation

$$
(b+c) f x^{2}-(b+c) F x+f^{2}=0 .
$$

Equation (3.5) has no real solution under the condition (3.1). Now in view of the fact that $\left[0, \frac{F}{f}\right]$ is an invariant interval for $\mathrm{H}$ and so for $\mathrm{H}_{1}$, an application of Theorem 1.1 completes the proof.

Remark 3.2. By Theorem 2.1 the equilibrium point $\bar{x}$ is locally asymptotically stable if

$$
f^{3}>\frac{c^{3} F^{2}}{(b+2 c)^{2}},
$$

and by Theorem 3.1 the equilibrium point $\bar{x}$ is globally asymptotically stable if the condition (3.1) holds. It can be shown that condition (3.1) implies (3.6), that is global asymptotic stability implies the local. We conjecture that the converse is true.

Conjecture 3.3. The equilibrium point $\bar{x}$ of (1.1) is globally asymptotically stable if it is locally asymptotically stable. 


\section{Naimark-Sacker bifurcation for equation (1.1)}

In this section we consider bifurcation of a fixed point of a map associated to (1.1) in the case where the eigenvalues are complex conjugate numbers on the unit circle.

The Naimark-Sacker bifurcation occurs for a discrete system depending on a parameter, $\lambda$, with a fixed point whose Jacobian matrix has a pair of complex conjugate eigenvalues $\mu(\lambda), \bar{\mu}(\lambda)$ which cross the unit circle transversally at $\lambda=\lambda_{0}$

For the sake of completeness we include the Naimark-Sacker bifurcation theorem of the interior fixed point. See $[3,8,13,14,16]$ for detailed description and especially [13] for the detailed proof of the result.

Theorem 4.1 (Poincare-Andronov-Hopf bifurcation or Naimark-Sacker bifurcation for maps). Let

$$
F: \mathbb{R} \times \mathbb{R}^{2} \rightarrow \mathbb{R}^{2}: \quad(\lambda, x) \rightarrow F(\lambda, x),
$$

be a $\mathrm{C}^{4}$ map depending on real parameter $\lambda$ satisfying the following conditions:

(i) $F(\lambda, 0)=0$ for $\lambda$ near some fixed $\lambda_{0}$;

(ii) $\operatorname{DF}(\lambda, 0)$ has two non-real eigenvalues $\mu(\lambda)$ and $\overline{\mu(\lambda)}$ for $\lambda$ near $\lambda_{0}$ with $\left|\mu\left(\lambda_{0}\right)\right|=1$;

(iii) $\frac{\mathrm{d}}{\mathrm{d} \lambda}|\mu(\lambda)|=\mathrm{d}\left(\lambda_{0}\right) \neq 0$ at $\lambda=\lambda_{0}$;

(iv) $\mu^{k}\left(\lambda_{0}\right) \neq 1$, for $k=1,2,3,4$.

Then there is a smooth $\lambda$-dependent change of coordinate bringing $f$ into the form

$$
F(\lambda, x)=\mathcal{F}(\lambda, x)+O\left(\|x\|^{5}\right),
$$

and there are smooth functions $\mathrm{a}(\lambda), \mathrm{b}(\lambda)$ and $\omega(\lambda)$ so that in polar coordinates the function $\mathcal{F}(\lambda, x)$ is given by

$$
\left(\begin{array}{l}
r \\
\theta
\end{array}\right)=\left(\begin{array}{c}
|\mu(\lambda)| r-a(\lambda) r^{3} \\
\theta+\omega(\lambda)+b(\lambda) r^{2}
\end{array}\right)
$$

If $\mathrm{a}\left(\lambda_{0}\right)>0$, then there is a neighborhood $\mathrm{U}$ of the origin and $a \delta>0$ such that for $\left|\lambda-\lambda_{0}\right|<\delta$ and $x_{0} \in \mathrm{U}$, then $\omega$-limit set of $x_{0}$ is the origin if $\lambda<\lambda_{0}$ and belongs to a closed invariant $C^{1}$ curve $\Gamma(\lambda)$ encircling the origin if $\lambda>\lambda_{0}$. Furthermore, $\Gamma\left(\lambda_{0}\right)=0$.

If $\mathrm{a}\left(\lambda_{0}\right)<0$, then there is a neighborhood $\mathrm{U}$ of the origin and $a \delta>0$ such that for $\left|\lambda-\lambda_{0}\right|<\delta$ and $x_{0} \in \mathrm{U}$, then $\alpha$-limit set of $x_{0}$ is the origin if $\lambda>\lambda_{0}$ and belongs to a closed invariant $C^{1}$ curve $\Gamma(\lambda)$ encircling the origin if $\lambda<\lambda_{0}$. Furthermore, $\Gamma\left(\lambda_{0}\right)=0$.

Consider a general map $F(\lambda, x)$ that has a fixed point at the origin with complex eigenvalues $\mu(\lambda)=$ $\alpha(\lambda)+i \beta(\lambda)$ and $\overline{\mu(\lambda)}=\alpha(\lambda)-i \beta(\lambda)$ satisfying $(\alpha(\lambda))^{2}+(\beta(\lambda))^{2}=1$ and $\beta(\lambda) \neq 0$. By transforming the linear part of such a map into Jordan normal form, we may assume $F$ to have the following form near the origin

$$
F(\lambda, x)=\left(\begin{array}{rr}
\alpha(\lambda) & -\beta(\lambda) \\
\beta(\lambda) & \alpha(\lambda)
\end{array}\right)\left(\begin{array}{l}
x_{1} \\
x_{2}
\end{array}\right)+\left(\begin{array}{l}
g_{1}\left(\lambda, x_{1}, x_{2}\right) \\
g_{2}\left(\lambda, x_{1}, x_{2}\right)
\end{array}\right)
$$

Then the coefficient $a\left(\lambda_{0}\right)$ of the cubic term in (4.1) in polar coordinates is equal to

$$
a\left(\lambda_{0}\right)=\operatorname{Re}\left(\frac{\left(1-2 \mu\left(\lambda_{0}\right)\right){\overline{\mu\left(\lambda_{0}\right)}}^{2}}{1-\mu\left(\lambda_{0}\right)} \xi_{11} \xi_{20}\right)+\frac{1}{2}\left|\xi_{11}\right|^{2}+\left|\xi_{02}\right|^{2}-\operatorname{Re}\left(\overline{\mu\left(\lambda_{0}\right)} \xi_{21}\right),
$$

where

$$
\xi_{20}=\frac{1}{8}\left(\frac{\partial^{2} g_{1}(0,0)}{\partial x_{1}^{2}}-\frac{\partial^{2} g_{1}(0,0)}{\partial x_{2}^{2}}+2 \frac{\partial^{2} g_{2}(0,0)}{\partial x_{1} \partial x_{2}}+i\left(\frac{\partial^{2} g_{2}(0,0)}{\partial x_{1}^{2}}-\frac{\partial^{2} g_{2}(0,0)}{\partial x_{2}^{2}}-2 \frac{\partial^{2} g_{1}(0,0)}{\partial x_{1} \partial x_{2}}\right)\right),
$$




$$
\begin{aligned}
& \xi_{11}=\frac{1}{4}\left(\frac{\partial^{2} g_{1}(0,0)}{\partial x_{1}^{2}}+\frac{\partial^{2} g_{1}(0,0)}{\partial x_{2}^{2}}+i\left(\frac{\partial^{2} g_{2}(0,0)}{\partial x_{1}^{2}}+\frac{\partial^{2} g_{2}(0,0)}{\partial x_{2}^{2}}\right)\right), \\
& \xi_{02}=\frac{1}{8}\left(\frac{\partial^{2} g_{1}(0,0)}{\partial x_{1}^{2}}-\frac{\partial^{2} g_{1}(0,0)}{\partial x_{2}^{2}}-2 \frac{\partial^{2} g_{2}(0,0)}{\partial x_{1} \partial x_{2}}+i\left(\frac{\partial^{2} g_{2}(0,0)}{\partial x_{1}^{2}}-\frac{\partial^{2} g_{2}(0,0)}{\partial x_{2}^{2}}+2 \frac{\partial^{2} g_{1}(0,0)}{\partial x_{1} \partial x_{2}}\right)\right),
\end{aligned}
$$

and

$$
\xi_{21}=\frac{1}{16}\left(\frac{\partial^{3} g_{1}}{\partial x_{1}^{3}}+\frac{\partial^{3} g_{1}}{\partial x_{1} \partial x_{2}^{2}}+\frac{\partial^{3} g_{2}}{\partial x_{1}^{2} \partial x_{2}}+\frac{\partial^{3} g_{2}}{\partial x_{2}^{3}}+i\left(\frac{\partial^{3} g_{2}}{\partial x_{1}^{3}}+\frac{\partial^{3} g_{2}}{\partial x_{1} \partial x_{2}^{2}}-\frac{\partial^{3} g_{1}}{\partial x_{1}^{2} \partial x_{2}}-\frac{\partial^{3} g_{1}}{\partial x_{2}^{3}}\right)\right) .
$$

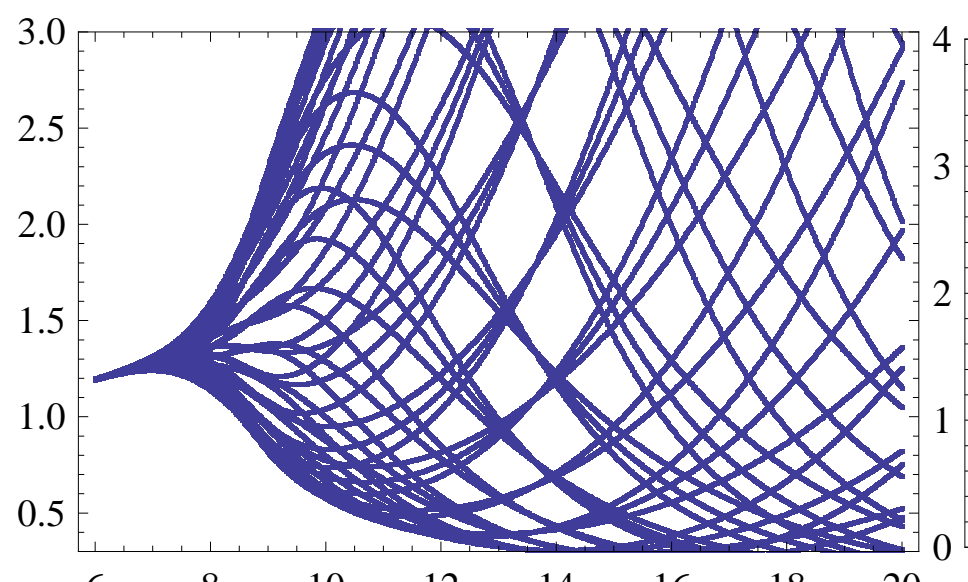

$\begin{array}{llllllll}6 & 8 & 10 & 12 & 14 & 16 & 18 & 20\end{array}$

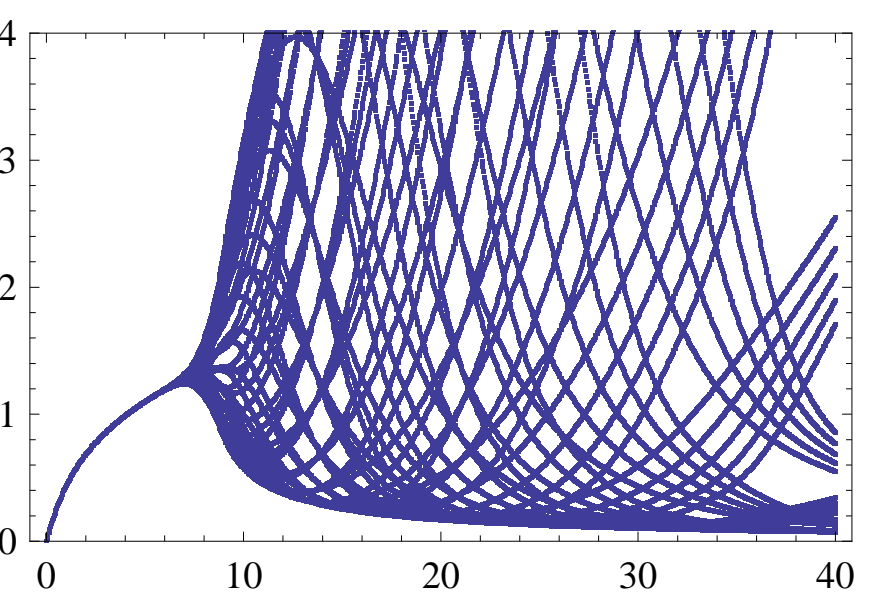

Figure 1: Bifurcation diagrams in $(\mathrm{F}, \mathrm{x})$ plane for $\mathrm{b}=1.2, \mathrm{c}=1, \mathrm{f}=1.9$, generated by Dynamica 3 [8].

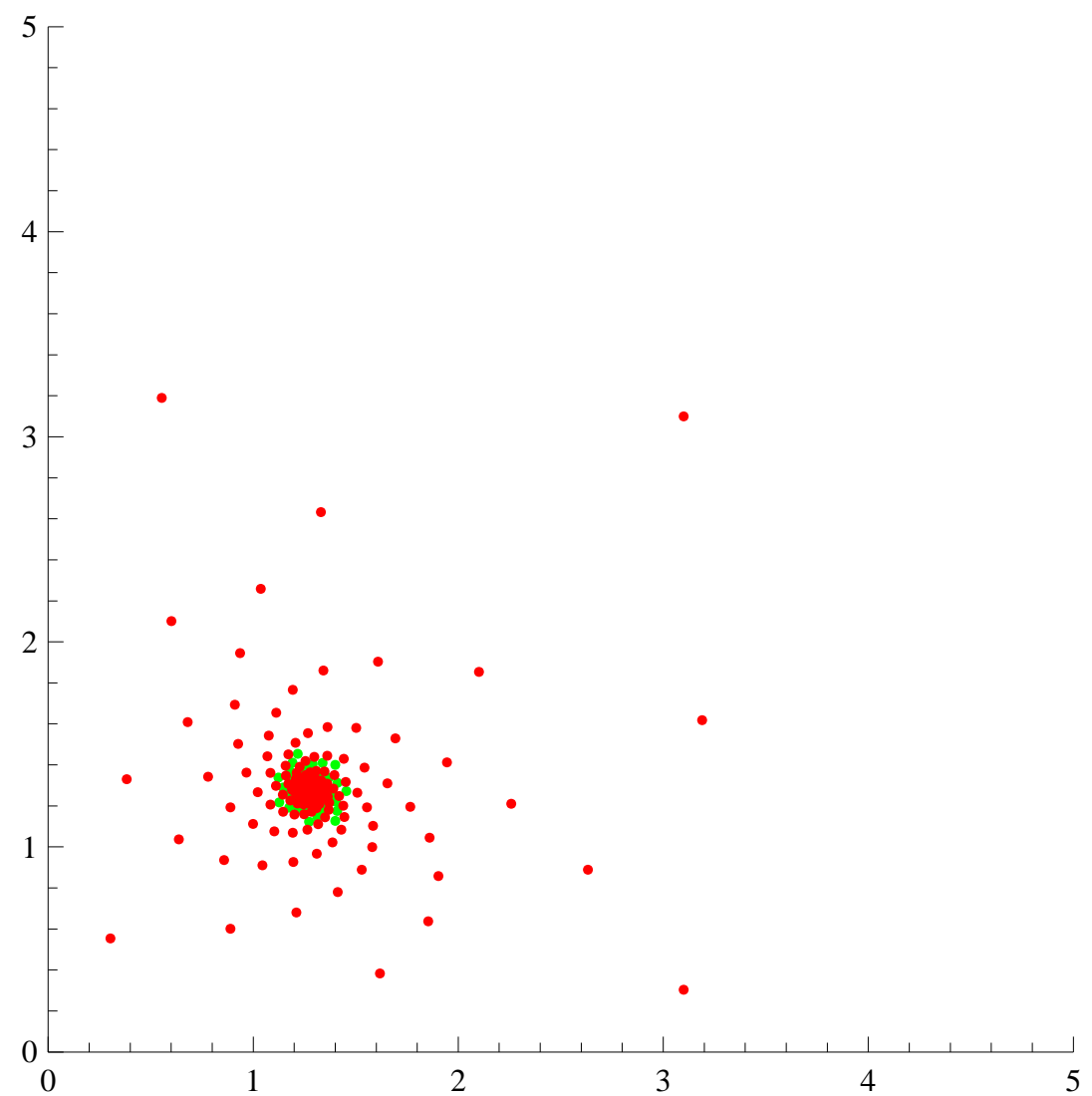

Figure 2: Phase portraits when $F=7<F_{0}\left(c=1, f=1.9, b=1.2<2 / 3+\cos ((1 / 3) \arctan \sqrt{107} / 53), x_{-1}=x_{0}=1.4\right.$ (green), $x_{-1}=x_{0}=3.1($ red), generated by Dynamica $3[8]$. 


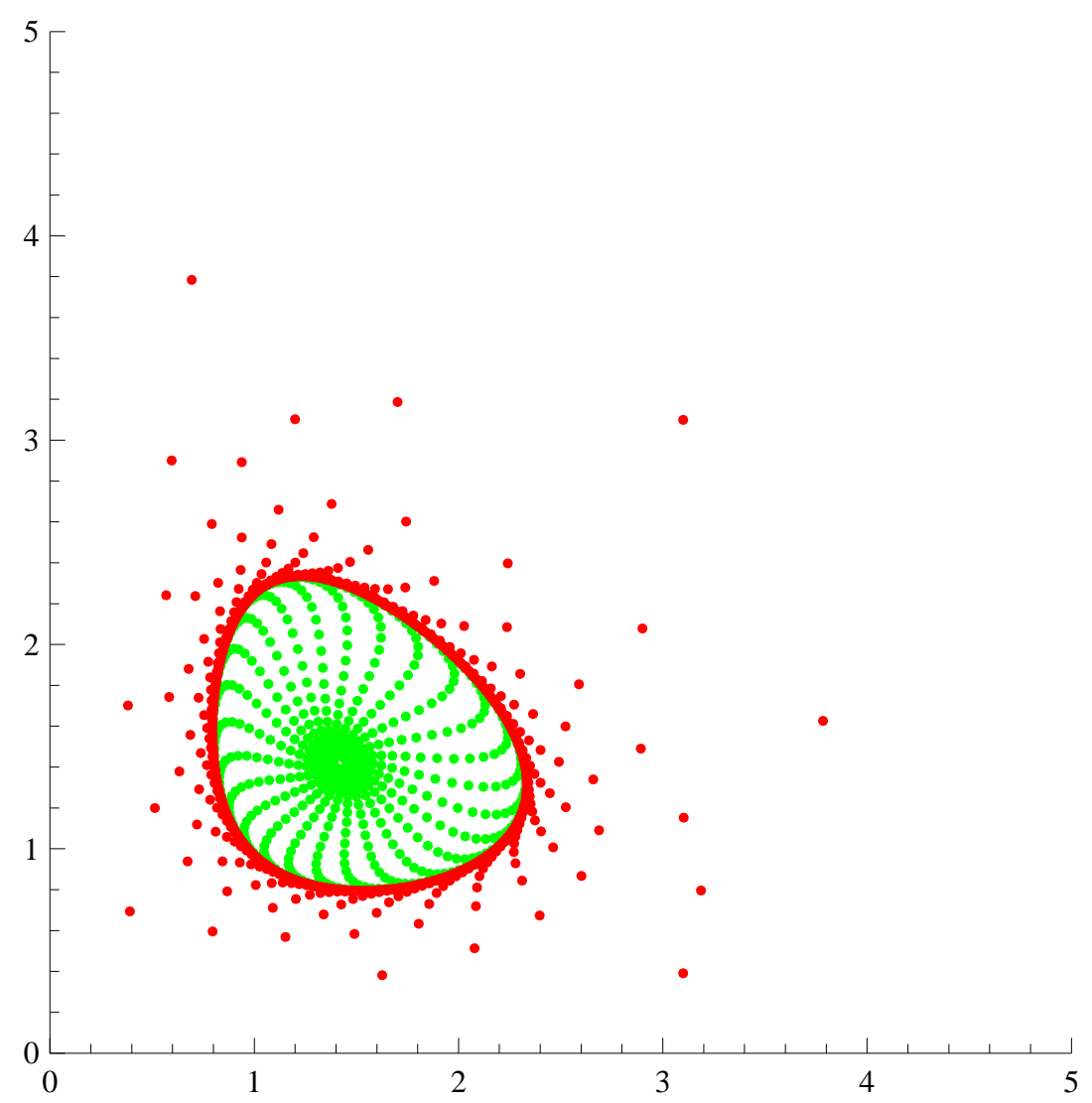

Figure 3: Phase portraits when $F=9>F_{0}\left(c=1, f=1.9, b=1.2<2 / 3+\cos ((1 / 3) \arctan \sqrt{107} / 53), x_{-1}=x_{0}=1.4(\right.$ green $)$ $x_{-1}=x_{0}=3.1$ (red), generated by Dynamica 3 [8].

Theorem 4.2. Assume that $\mathrm{b}, \mathrm{c}, \mathrm{f}>0$ and

$$
F_{0}=\frac{f \sqrt{f}(b+2 c)}{c \sqrt{c}} \text { and } \bar{x}=\frac{\sqrt{f}}{\sqrt{c}}
$$

(i) If $0<\mathrm{b}<\left(\frac{2}{3}+4 \cos \frac{1}{3}\left(\arctan \frac{1}{53} \sqrt{107}\right)\right) \mathrm{c}$, then there is a neighborhood $\mathrm{U}$ of the equilibrium point $\overline{\mathrm{x}}$ and a $\rho>0$ such that for $\left|\mathrm{F}-\mathrm{F}_{0}\right|<\rho$ and $x_{0}, x_{-1} \in \mathrm{U}$, then $\omega$-limit set of solution of (1.1), with initial condition $x_{0}, x_{-1}$ is the equilibrium point $\bar{x}$ if $\mathrm{F}<\mathrm{F}_{0}$ and belongs to a closed invariant $\mathrm{C}^{1}$ curve $\Gamma\left(\mathrm{F}_{0}\right)$ encircling the equilibrium point $\overline{\mathrm{x}}$ if $\mathrm{F}>\mathrm{F}_{0}$. Furthermore, $\Gamma\left(\mathrm{F}_{0}\right)=0$.

(ii) If $\mathrm{b}>\left(\frac{2}{3}+4 \cos \frac{1}{3}\left(\arctan \frac{1}{53} \sqrt{107}\right)\right) \mathrm{c}$, then there is a neighborhood $\mathrm{U}$ of the equilibrium point $\overline{\mathrm{x}}$ and $a$ $\rho>0$ such that for $\left|\mathrm{F}-\mathrm{F}_{0}\right|<\rho$ and $\mathrm{x}_{0}, \mathrm{x}_{-1} \in \mathrm{U}$, then $\alpha$-limit set of $\mathrm{x}_{0}, \mathrm{x}_{-1}$ is the equilibrium point $\overline{\mathrm{x}}$ if $\mathrm{F}>\mathrm{F}_{0}$ and belongs to a closed invariant $\mathrm{C}^{1}$ curve $\Gamma\left(\mathrm{F}_{0}\right)$ encircling the equilibrium point $\overline{\mathrm{x}}$ if $\mathrm{F}<\mathrm{F}_{0}$. Furthermore, $\Gamma\left(\mathrm{F}_{0}\right)=0$.

Proof. In order to apply Theorem 4.1 we make a change of variable $y_{n}=x_{n}-\bar{x}$. Then, the new equation is given by

$$
y_{n+1}=\frac{F}{b\left(y_{n}+\bar{x}\right)\left(y_{n-1}+\bar{x}\right)+c\left(y_{n-1}+\bar{x}\right)^{2}+f}-\bar{x}
$$

Set

$$
u_{n}=y_{n-1} \text { and } v_{n}=y_{n}, \quad \text { for } n=0,1, \cdots,
$$


and write

$$
\left.\begin{array}{l}
u_{n+1}=v_{n}, \\
v_{n+1}=\frac{F}{b\left(v_{n}+\bar{x}\right)\left(u_{n}+\bar{x}\right)+c\left(u_{n}+\bar{x}\right)^{2}+f}-\bar{x},
\end{array}\right\}
$$

where

$$
F(u, v)=\left(\frac{v}{b(v+\bar{x})(u+\bar{x})+c(u+\bar{x})^{2}+f}-\bar{x}\right) .
$$

Then $F(u, v)$ has the unique fixed point $(0,0)$. The Jacobian matrix of $F(u, v)$ is given by

$$
J_{F}(u, v)=\left(\begin{array}{cc}
0 & 1 \\
\frac{-F(b(v+\bar{x})+2 c(u+\bar{x}))}{\left(b(v+\bar{x})(u+\bar{x})+c(u+\bar{x})^{2}+f\right)^{2}} & \frac{-F b(u+\bar{x})}{\left(b(v+\bar{x})(u+\bar{x})+c(u+\bar{x})^{2}+f\right)^{2}}
\end{array}\right),
$$

and its value at the zero equilibrium is

$$
J_{0}=J_{F}(0,0)=\left(\begin{array}{cc}
0 & 1 \\
\frac{-F(b+2 c) \bar{x}}{\left(b \bar{x}^{2}+c \bar{x}^{2}+f\right)^{2}} & \frac{-F b \bar{x}}{\left(b \bar{x}^{2}+c \bar{x}^{2}+f\right)^{2}}
\end{array}\right)=\left(\begin{array}{cc}
0 & 1 \\
\frac{-(b+2 c)}{F} \bar{x}^{3} & \frac{-b}{F} \bar{x}^{3}
\end{array}\right) .
$$

The eigenvalues are $\mu(F)$ and $\overline{\mu(F)}$ where

$$
\mu(F)=\frac{-b \bar{x}^{3} \pm i \sqrt{\bar{x}^{3}\left(4 F(b+2 c)-b^{2} \bar{x}^{3}\right)}}{2 F},
$$

and

$$
4 F(b+2 c)-b^{2} \bar{x}^{3}=4\left((b+c) \bar{x}^{3}+f \bar{x}\right)(b+2 c)-b^{2} \bar{x}^{3}>0
$$

Then we have that

$$
F\left(\begin{array}{l}
u \\
v
\end{array}\right)=\left(\begin{array}{cc}
0 & 1 \\
\frac{-(b+2 c)}{F} \bar{x}^{3} & \frac{-b}{F} \bar{x}^{3}
\end{array}\right)\left(\begin{array}{l}
u \\
v
\end{array}\right)+\left(\begin{array}{c}
f_{1}(F, u, v) \\
f_{2}(F, u, v)
\end{array}\right)
$$

and

$$
\begin{aligned}
& f_{1}(F, u, v)=0, \\
& f_{2}(F, u, v)=\frac{F}{b(v+\bar{x})(u+\bar{x})+c(u+\bar{x})^{2}+f}+\frac{(b+2 c) u}{F} \bar{x}^{3}+\frac{b v}{F} \bar{x}^{3}-\bar{x} .
\end{aligned}
$$

Let

$$
F_{0}=\frac{f \sqrt{f}(b+2 c)}{c \sqrt{c}}
$$

For $\mathrm{F}=\mathrm{F}_{0}$ we obtain

$$
\bar{x}=\frac{\sqrt{f}}{\sqrt{c}}, \quad \bar{\chi}^{3}=\frac{F_{0}}{b+2 c} .
$$

The eigenvalues of $J_{0}$ are $\mu\left(F_{0}\right)$ and $\overline{\mu\left(F_{0}\right)}$ where

$$
\mu\left(F_{0}\right)=\frac{-b+i \sqrt{(3 b+4 c)(b+4 c)}}{2(b+2 c)}, \quad\left|\mu\left(F_{0}\right)\right|=1 .
$$

The eigenvectors corresponding to $\mu\left(\mathrm{F}_{0}\right)$ and $\overline{\mu\left(\mathrm{F}_{0}\right)}$ are $v\left(\mathrm{~F}_{0}\right)$ and $\overline{v\left(\mathrm{~F}_{0}\right)}$ where

$$
v\left(F_{0}\right)=\left(\frac{-b-i \sqrt{(3 b+4 c)(b+4 c)}}{2(b+2 c)}, 1\right) .
$$


Then

$$
\begin{aligned}
& \left|\mu\left(F_{0}\right)\right|=1, \\
& \mu^{2}\left(F_{0}\right)=-\frac{8 b c+b^{2}+8 c^{2}}{2(b+2 c)^{2}}-i b \frac{\sqrt{(3 b+4 c)(b+4 c)}}{2(b+2 c)^{2}}, \\
& \mu^{3}\left(F_{0}\right)=b \frac{6 b c+b^{2}+6 c^{2}}{(b+2 c)^{3}}-i \frac{2 c(b+c) \sqrt{(3 b+4 c)(b+4 c)}}{(b+2 c)^{3}}, \\
& \mu^{4}\left(F_{0}\right)=\frac{64 b c^{3}+32 b^{2} c^{2}-b^{4}+32 c^{4}}{2(b+2 c)^{4}}+i \frac{\left(8 b c+b^{2}+8 c^{2}\right) b \sqrt{(3 b+4 c)(b+4 c)}}{2(b+2 c)^{4}},
\end{aligned}
$$

and $\mu^{k}\left(F_{0}\right) \neq 1$ for $k=1,2,3,4$.

For $F=F_{0}$ and $\bar{x}=\frac{\sqrt{f}}{\sqrt{c}}$ we get

$$
F\left(\begin{array}{l}
u \\
v
\end{array}\right)=\left(\begin{array}{cc}
0 & 1 \\
-1 & \frac{-b}{b+2 c}
\end{array}\right)\left(\begin{array}{l}
u \\
v
\end{array}\right)+\left(\begin{array}{l}
h_{1}(u, v) \\
h_{2}(u, v)
\end{array}\right)
$$

and

$$
\begin{aligned}
h_{1}(u, v) & =f_{1}\left(F_{0}, u, v\right)=0, \\
h_{2}(u, v) & =f_{2}\left(F_{0}, u, v\right) \\
& =\frac{\sqrt{c}\left(2 c^{\frac{5}{2}} u^{3}+b^{2} \sqrt{f} u^{2}+b^{2} \sqrt{f} v^{2}+2 c^{2} \sqrt{f} u^{2}+b c^{\frac{3}{2}} u^{3}+b^{2} \sqrt{c} u v^{2}+b^{2} \sqrt{c} u^{2} v+3 b c \sqrt{f} u^{2}+3 b c^{\frac{3}{2}} u^{2} v+b^{2} \sqrt{f} u v+2 b c \sqrt{f} u v\right)}{(b+2 c)\left(c^{2} u^{2}+b f+2 c f+2 c^{\frac{3}{2}} \sqrt{f} u+b \sqrt{c} \sqrt{f} u+b \sqrt{c} \sqrt{f} v+b c u v\right)} .
\end{aligned}
$$

Hence, for $F=F_{0}$ system (4.7) takes the form

$$
\left(\begin{array}{l}
u_{n+1} \\
v_{n+1}
\end{array}\right)=\left(\begin{array}{cc}
0 & 1 \\
-1 & \frac{-b}{b+2 c}
\end{array}\right)\left(\begin{array}{l}
u_{n} \\
v_{n}
\end{array}\right)+\left(\begin{array}{l}
h_{1}\left(u_{n}, v_{n}\right) \\
h_{2}\left(u_{n}, v_{n}\right)
\end{array}\right) .
$$

For

$$
\left(\begin{array}{l}
u_{n} \\
v_{n}
\end{array}\right)=P\left(\begin{array}{l}
\xi_{n} \\
\eta_{n}
\end{array}\right)
$$

where

$$
P=\left(\begin{array}{cc}
\frac{-b}{2(b+2 c)} & \frac{\sqrt{(3 b+4 c)(b+4 c)}}{2(b+2 c)} \\
1 & 0
\end{array}\right), \quad P^{-1}=\left(\begin{array}{cc}
0 & 1 \\
\frac{2(b+2 c)}{\sqrt{(3 b+4 c)(b+4 c)}} & \frac{b}{\sqrt{(3 b+4 c)(b+4 c)}}
\end{array}\right),
$$

system (4.7) is equivalent to its normal form

$$
\left(\begin{array}{c}
\xi_{n+1} \\
\eta_{n+1}
\end{array}\right)=\left(\begin{array}{cc}
-\frac{b}{2(b+2 c)} & -\frac{\sqrt{(3 b+4 c)(b+4 c)}}{2(b+2 c)} \\
\frac{\sqrt{(3 b+4 c)(b+4 c)}}{2(b+2 c)} & -\frac{b}{2(b+2 c)}
\end{array}\right)\left(\begin{array}{c}
\xi_{n} \\
\eta_{n}
\end{array}\right)+P^{-1} H\left(P\left(\begin{array}{c}
\xi_{n} \\
\eta_{n}
\end{array}\right)\right),
$$

where

$$
H\left(\begin{array}{l}
u \\
v
\end{array}\right):=\left(\begin{array}{l}
h_{1}(u, v) \\
h_{2}(u, v)
\end{array}\right) .
$$

Let

$$
\mathrm{G}\left(\begin{array}{l}
u \\
v
\end{array}\right)=\left(\begin{array}{l}
g_{1}(u, v) \\
g_{2}(u, v)
\end{array}\right)=P^{-1} H\left(P\left(\begin{array}{l}
u \\
v
\end{array}\right)\right) .
$$

By straightforward calculation we obtain that

$$
g_{1}(u, v)=\frac{\sqrt{c}}{b+2 c} \wedge(u, v),
$$


with

$$
\Lambda(u, v)=\frac{(b+2 c) c \sqrt{c}(h(u, v))^{3}+((b+c)(b+2 c) \sqrt{f}+(b+3 c) b u \sqrt{c})(h(u, v))^{2}+b u(b u \sqrt{c}+(b+2 c) \sqrt{f}) h(u, v)+b^{2} u^{2} \sqrt{f}}{c^{2}(h(u, v))^{2}+(b c u+(b+2 c) \sqrt{c f}) h(u, v)+b u \sqrt{c f}+(b+2 c) f},
$$

where

$$
h(u, v)=\frac{v \sqrt{(3 b+4 c)(b+4 c)}-b u}{2(b+2 c)}
$$

and

$$
g_{2}(u, v)=\frac{b}{\sqrt{(3 b+4 c)(b+4 c)}} g_{1}(u, v) .
$$

Another straightforward calculation gives

$$
\begin{aligned}
& \frac{\partial^{2} g_{1}(0,0)}{\partial u^{2}}=\frac{b^{2} \sqrt{c}(3 b+5 c)}{2 \sqrt{f}(b+2 c)^{3}}, \\
& \frac{\partial^{2} g_{1}(0,0)}{\partial u \partial v}=\frac{b c^{\frac{3}{2}} \sqrt{(3 b+4 c)(b+4 c)}}{2 \sqrt{f}(b+2 c)^{3}}, \\
& \frac{\partial^{2} g_{1}(0,0)}{\partial v^{2}}=\frac{\sqrt{c}(b+4 c)(3 b+4 c)(b+c)}{2 \sqrt{f}(b+2 c)^{3}}, \\
& \frac{\partial^{3} g_{1}(0,0)}{\partial u^{3}}=-\frac{3 b^{3} c(5 b+8 c)}{4 f(b+2 c)^{4}}, \\
& \frac{\partial^{3} g_{1}(0,0)}{\partial u \partial v^{2}}=\frac{b^{2} c(3 b+4 c)(b+4 c)}{4 f(b+2 c)^{4}}, \\
& \frac{\partial^{3} g_{1}(0,0)}{\partial u^{2} \partial v}=-\frac{b^{2} c(3 b+8 c) \sqrt{16 b c+3 b^{2}+16 c^{2}}}{4 f(b+2 c)^{4}}, \\
& \frac{\partial^{3} g_{1}(0,0)}{\partial v^{3}}=-\frac{3 b c\left(\sqrt{16 b c+3 b^{2}+16 c^{2}}\right)^{3}}{4 f(b+2 c)^{4}} .
\end{aligned}
$$

By using (4.2), (4.3), (4.4), (4.5), and (4.6) for $\lambda_{0}=F_{0}, x_{1}=u$ and $x_{2}=v$ we obtain

$$
\begin{aligned}
& \xi_{11}=\frac{\sqrt{c}\left(3 b^{2}+6 b c+4 c^{2}\right)}{4 \sqrt{f}(b+2 c)^{2}}\left(1+i \frac{b}{\sqrt{(3 b+4 c)(b+4 c)}}\right), \\
& \xi_{20}=\frac{1}{8}\left(\frac{-2(\sqrt{c})^{3}(3 b+2 c)}{(b+2 c)^{2} \sqrt{f}}+i\left(\frac{-b(\sqrt{c})^{3}\left(32 b c+10 b^{2}+24 c^{2}\right)}{\sqrt{16 b c+3 b^{2}+16 c^{2} \sqrt{f}(b+2 c)^{3}}}\right)\right), \\
& \xi_{20}=-\frac{c^{\frac{3}{2}}\left((3 b+2 c) \sqrt{3 b^{2}+16 b c+16 c^{2}}+i b(6 c+5 b)\right)}{4 \sqrt{f}(b+2 c)^{2} \sqrt{3 b^{2}+16 b c+16 c^{2}}}, \\
& \xi_{02}=\frac{-c^{\frac{3}{2}}}{\sqrt{f}(b+2 c)^{3}}\left((b+c)^{2}+i\left(\frac{b\left(b^{2}-2 c^{2}\right)}{2 \sqrt{3 b^{2}+16 b c+16 c^{2}}}\right)\right), \\
& \xi_{21}=\frac{b c}{8 f(b+2 c)^{4}}\left(-b\left(8 b c+3 b^{2}+4 c^{2}\right)+i\left(\frac{(b+2 c)\left(80 b c^{2}+38 b^{2} c+3 b^{3}+48 c^{3}\right)}{\sqrt{3 b^{2}+16 b c+16 c^{2}}}\right)\right), \\
& \frac{\left(1-2 \mu\left(F_{0}\right)\right) \frac{1\left(F_{0}\right)^{2}}{1-\mu\left(F_{0}\right)}=\frac{-(3 b+4 c)\left(10 b c+b^{2}+12 c^{2}\right)+i\left(10 b c+5 b^{2}+4 c^{2}\right) \sqrt{3 b^{2}+16 b c+16 c^{2}}}{2(b+2 c)^{2}(3 b+4 c)},}{2 f(b+2 c)^{4}}\left(\frac{c^{2}\left(20 b c^{2}+12 b^{2} c+b^{3}+8 c^{3}\right)}{2(3 b+4 c)(b+4 c)}+i \frac{b c^{2}(b+c)}{\sqrt{16 b c+3 b^{2}+16 c^{2}}}\right), \\
& \xi_{11} \xi_{20}=\frac{-\left(6 b c+3 b^{2}+4 c^{2}\right)}{2},
\end{aligned}
$$




$$
\begin{aligned}
& \operatorname{Re}\left(\frac{\left(1-2 \mu\left(F_{0}\right)\right){\overline{\mu\left(F_{0}\right)}}^{2}}{1-\mu\left(F_{0}\right)} \xi_{11} \xi_{20}\right)=\frac{c^{2}\left(6 b c+3 b^{2}+4 c^{2}\right)\left(26 b c+11 b^{2}+12 c^{2}\right)}{8 f(b+2 c)^{3}(b+4 c)(3 b+4 c)}, \\
& \frac{1}{2}\left|\xi_{11}\right|^{2}=\frac{c\left(3 b^{2}+6 b c+4 c^{2}\right)^{2}}{8 f(b+2 c)^{2}(3 b+4 c)(b+4 c)} \\
& \xi_{02}=\frac{-c^{\frac{3}{2}}}{\sqrt{f}(b+2 c)^{3}}\left((b+c)^{2}+i\left(\frac{b\left(b^{2}-2 c^{2}\right)}{2 \sqrt{3 b^{2}+16 b c+16 c^{2}}}\right)\right), \\
& \left|\xi_{02}\right|^{2}=\frac{c^{3}\left(28 b c^{2}+34 b^{2} c+13 b^{3}+8 c^{3}\right)}{4 f(b+2 c)^{3}(3 b+4 c)(b+4 c)}, \\
& \operatorname{Re}\left(\overline{\mu\left(F_{0}\right)} \xi_{21}\right)=\frac{b c}{8 f(b+2 c)^{4}}\left(\frac{b^{2}\left(8 b c+3 b^{2}+4 c^{2}\right)}{2(b+2 c)}+\frac{\left(80 b c^{2}+38 b^{2} c+3 b^{3}+48 c^{3}\right)}{2}\right)=\frac{b c\left(14 b c+3 b^{2}+12 c^{2}\right)}{8 f(b+2 c)^{3}} \text {, } \\
& \xi_{21}=\frac{b c}{8 f(b+2 c)^{4}}\left(-b\left(8 b c+3 b^{2}+4 c^{2}\right)+i\left(\frac{(b+2 c)\left(80 b c^{2}+38 b^{2} c+3 b^{3}+48 c^{3}\right)}{\sqrt{3 b^{2}+16 b c+16 c^{2}}}\right)\right), \\
& a\left(F_{0}\right)=\frac{c^{2}\left(-3 b^{3}+6 b^{2} c+32 b c^{2}+24 c^{3}\right)}{8 f(b+2 c)^{3}(3 b+4 c)} \text {. }
\end{aligned}
$$

So,

$$
a\left(F_{0}\right)=0 \Leftrightarrow-3 b^{3}+6 b^{2} c+32 b c^{2}+24 c^{3}=0 .
$$

By substituting $b=k c$ we obtain

$$
-3(k c)^{3}+6(k c)^{2} c+32(k c) c^{2}+24 c^{3}=0,
$$

i.e.,

$$
k^{3}-2 k^{2}-\frac{32}{3} k-8=0 .
$$

By using Cardano's substitution $k=y+\frac{2}{3}$, we obtain

$$
y^{3}-12 y-\frac{424}{27}=0
$$

with the corresponding positive solution

$$
y=\frac{1}{3} \sqrt[3]{212+4 i \sqrt{107}}+\frac{1}{3} \sqrt[3]{212-4 i \sqrt{107}}
$$

The corresponding angle and modulus are

$$
\begin{aligned}
\tan \alpha & =\frac{\sqrt{107}}{53}, \\
\alpha & =\arctan \frac{1}{53} \sqrt{107}, \\
r & =\sqrt{(212)^{2}+(16)(107)}=216,
\end{aligned}
$$

for which we obtain

$$
y=\frac{1}{3}\left(6\left(\cos \frac{\alpha}{3}+i \sin \frac{\alpha}{3}\right)\right)+\frac{1}{3}\left(6\left(\cos \frac{\alpha}{3}-i \sin \frac{\alpha}{3}\right)\right)=4 \cos \frac{1}{3} \alpha,
$$

and

$$
k=y+\frac{2}{3}=\frac{2}{3}+4 \cos \frac{1}{3} \alpha
$$


Hence,

$$
a\left(F_{0}\right)=0 \quad \text { for } \quad b=\left(\frac{2}{3}+4 \cos \frac{1}{3} \arctan \frac{\sqrt{107}}{53}\right) c
$$

Now

$$
\mu(F)=\frac{-b \bar{x}^{3} \pm i \sqrt{\bar{x}^{3}\left(4 F(b+2 c)-b^{2} \bar{x}^{3}\right)}}{2 F}
$$

and so

$$
\mu(F) \overline{\mu(F)}=\frac{(b+2 c) x^{3}}{F} .
$$

Thus

$$
|\mu(F)|=\sqrt{\frac{(b+2 c) x^{3}}{F}} .
$$

Differentiating the equilibrium equation

$$
(b+c) x^{3}+f x-F=0,
$$

with respect to $F$ and solving for $\chi^{\prime}(F)$ we obtain

$$
\begin{aligned}
x^{\prime}(F) & =\frac{1}{3(b+c)(x(F))^{2}+f^{\prime}}, \quad x\left(F_{0}\right)=\sqrt{\frac{f}{c}} \\
x^{\prime}\left(F_{0}\right) & =\frac{1}{3(b+c) \frac{f}{c}+f}=\frac{c}{f(3 b+4 c)} .
\end{aligned}
$$

By substituting $x^{\prime}(F)$ in the expression

$$
\frac{d|\mu(F)|}{d F}=\frac{1}{2 \sqrt{\frac{(b+2 c) x^{3}}{F}}}\left(\frac{3(b+2 c) x^{2} x^{\prime}}{F}-\frac{(b+2 c) x^{3}}{F^{2}}\right), \quad x=x(F),
$$

we obtain that

$$
\frac{d|\mu(F)|}{d F}\left(F_{0}\right)=\frac{1}{2 \sqrt{\frac{(b+2 c)}{F_{0}} \cdot \frac{F_{0}}{(b+2 c)}}}\left(\frac{3(b+2 c)\left(\sqrt{\frac{f}{c}}\right)^{2} \frac{c}{f(3 b+4 c)}-1}{F_{0}}\right)
$$

and

$$
\frac{d|\mu(F)|}{d F}\left(F_{0}\right)=\frac{c}{F_{0}(3 b+4 c)}=\frac{c^{2} \sqrt{c}}{f \sqrt{f}(b+2 c)(3 b+4 c)}>0,
$$

which completes the proof of theorem.

The visual illustration of Theorem 4.1 is given in Figures 1-3. Figure 1 shows the bifurcation diagram for a parameter range where the Naimark-Sacker bifurcation takes the place. Figures 2 and 3 show the transition from the global asymptotic stability of the equilibrium to the existence of a periodic solution.

\section{Acknowledgment}

This work was partially supported by the grant of the Ministry of Education and Science of the Federation of Bosnia and Herzegovina. 


\section{References}

[1] A. M. Amleh, E. Camouzis, G. Ladas, On the dynamics of a rational difference equation, I, Int. J. Difference Equ., 3 (2008), 1-35. 1

[2] A. M. Amleh, E. Camouzis, G. Ladas, On the dynamics of a rational difference equation, II, Int. J. Difference Equ., 3 (2008), 195-225. 1

[3] J. K. Hale, H. Kocak, Dynamics and bifurcations, Texts in Applied Mathematics, Springer-Verlag, New York, (1991). 4

[4] E. A. Janowski, M. R. S. Kulenović, Attractivity and global stability for linearizable difference equations, Comput. Math. Appl., 57 (2009), 1592-1607. 1

[5] C. M. Kent, H. Sedaghat, Global attractivity in a quadratic-linear rational difference equation with delay, J. Difference Equ. Appl., 15 (2009), 913-925.

[6] C. M. Kent, H. Sedaghat, Global attractivity in a rational delay difference equation with quadratic terms, J. Difference Equ. Appl., 17 (2011), 457-466. 1

[7] M. R. S. Kulenović, G. Ladas, Dynamics of second order rational difference equations, With open problems and conjectures, Chapman \& Hall/CRC, Boca Raton, FL, (2001). 1

[8] M. R. S. Kulenović, O. Merino, Discrete dynamical systems and difference equations with Mathematica, Chapman \& Hall/CRC, Boca Raton, FL, (2002). 4, 1, 2, 3

[9] M. R. S. Kulenović, O. Merino, A global attractivity result for maps with invariant boxes, Discrete Contin. Dyn. Syst. Ser. B, 6 (2006), 97-110. 1

[10] M. R. S. Kulenović, O. Merino, Global bifurcation for discrete competitive systems in the plane, Discrete Contin. Dyn. Syst. Ser. B, 12 (2009), 133-149. 1

[11] M. R. S. Kulenović, O. Merino, Invariant manifolds for competitive discrete systems in the plane, Internat. J. Bifur. Chaos Appl. Sci. Engrg., 20 (2010), 2471-2486. 1

[12] M. R. S. Kulenović, E. Pilav, E. Silić, Naimark-Sacker bifurcation of a certain second order quadratic fractional difference equation, J. Math. Comput. Sci., 4 (2014), 1025-1043. 1

[13] Y. A. Kuznetsov, Elements of applied bifurcation theory, Second edition. Applied Mathematical Sciences, SpringerVerlag, New York, (1998). 4

[14] C. Robinson, Stability, symbolic dynamics, and chaos, Stud. Adv. Math. Boca Raton, CRC Press, FL, (1995). 4

[15] H. Sedaghat, Global behaviours of rational difference equations of orders two and three with quadratic terms, J. Difference Equ. Appl., 15 (2009), 215-224. 1

[16] S. Wiggins, Introduction to applied nonlinear dynamical systems and chaos, Second edition, Texts in Applied Mathematics, Springer-Verlag, New York, (2003). 4 